\title{
Acrocyanosis Presentation in Postural Orthostatic Tachycardia Syndrome
}

\author{
Jane Abou-Diab $^{\mathrm{a}}$, Dina Moubayed ${ }^{\mathrm{a}, \mathrm{b}}$, Danielle Taddeo ${ }^{\mathrm{a}}$, Olivier Jamoulle ${ }^{\mathrm{a}}$, Chantal Stheneur ${ }^{\mathrm{a}}$
}

\begin{abstract}
Postural orthostatic tachycardia syndrome (POTS) is a clinical chronic condition characterized by chronic fatigue and orthostatic symptoms associated with postural tachycardia. Early diagnosis and intervention can prevent significant functional consequences, although it can be very difficult to recognize. Two cases of POTS were presented in this article to help clinicians identify POTS more easily. Firstly, we present a 16-year-old adolescent female who was hospitalized for daily headaches, chronic fatigue, bilateral lower limbs discoloration and edema on standing position associated with orthostatic symptoms. All investigations were negative except for tilt table test, which showed heart rate increase of 80 beats per min, hypotension and discoloration of lower limbs, confirming POTS diagnosis. Non-pharmacological treatment showed no improvement. Midodrine was started because of $\beta$-blockers intolerance with only moderate response. Secondly, we present a 17-year-old patient diagnosed with benign hypermobility syndrome who was referred for recurring episodes of palpitations associated with heat, nausea, headache and vertigo for the past year. She also reported dizziness and lower limbs discoloration on standing position. Previous investigations were negative. A clinical diagnosis of POTS was made based on history. Non-pharmacologic treatments with hydration, increase of salt intake and regular exercise were tested. However, no improvement was shown. Symptoms were controlled by a propranolol trial. These cases relate a clinical sign that can be easily identified by a clinician attempting to diagnose POTS. Acrocyanosis can be unquestioned or ignored. Clinicians should be attentive to this condition, and attempt to diagnose its underlying causes. This can result in lowering unnecessary testing and interventions. Patients with dependent acrocyanosis should be closely evaluated for the diagnosis of POTS.
\end{abstract}

Keywords: Acrocyanosis; Adolescence; Orthostatic; Tachycardia

\section{Introduction}

Postural orthostatic tachycardia syndrome (POTS) was first

Manuscript submitted February 5, 2018, accepted February 20, 2018

${ }^{a}$ Centre Hospitalier Universitaire Sainte-Justine, 3175 Chemin Cote SainteCatherine, Montreal, QC H3T 1C5, Canada

${ }^{b}$ Corresponding Author: Dina Moubayed, Department of Pediatrics, Centre Hospitalier Universitaire Sainte-Justine, 3175 Chemin Cote Sainte-Catherine, Montreal, QC H3T 1C5, Canada. Email: moubayeddina@gmail.com

doi: https://doi.org/10.14740/ijcp293w described in 1993 in adults [1], then in teenagers in 1999 [2]. POTS is a chronic clinical condition characterized by fatigue and symptoms of orthostatic intolerance associated with postural tachycardia [3], despite maintenance of normal blood pressure [4]. It commonly begins several years after puberty $[3,5]$ and presents mostly in Caucasian females (4 to 5:1 ratio) $[6,7]$. The prevalence in adults is estimated at 170 per 100,000 [6], whereas its prevalence in pediatrics population remains uncertain. Because of recent literature detailing the variety of symptoms, the prevalence has become difficult to estimate [4]. To our knowledge, our cases are the first pediatric patients with POTS with dependant acrocyanosis as a main symptom to be reported.

\section{Case Reports}

\section{Case 1}

A 16-year-old adolescent female was known for having right long thoracic nerve neuropathy secondary to a traumatic humeral fracture 3 years ago, daily headaches and chronic fatigue for 6 months. She reported bilateral lower limbs discoloration and edema (Fig. 1a, b) associated with orthostatic symptoms for over 1 year. These symptoms appeared daily on standing position and disappeared within 5 min of being seated down. It generated poor school attendance and a cessation of all physical activity. The physical exam was normal. Blood panel, lower limbs Doppler, cerebral CT scan, electrocardiogram and cardiac ultrasound were all within normal limits. Holter measurement showed sinus rhythm with a rate varying from 47 to $158 \mathrm{bpm}$. Tilt table test showed an increasing in heart rate (HR) from 70 to $150 \mathrm{bpm}$ associated with hypotension and discoloration of lower limbs, thus confirming POTS diagnosis. Non-pharmacological treatment was recommended. Two different $\beta$-blockers were tried because of the persisting symptoms. They were both stopped due to significant bradycardia. Midodrine was started with a moderate response. The patient was also referred to a psychologist.

\section{Case 2}

A 17-year-old female was referred for recurring episodes of palpitations associated with a heat sensation, nausea, headaches and vertigo for the past year. These episodes occurred up 

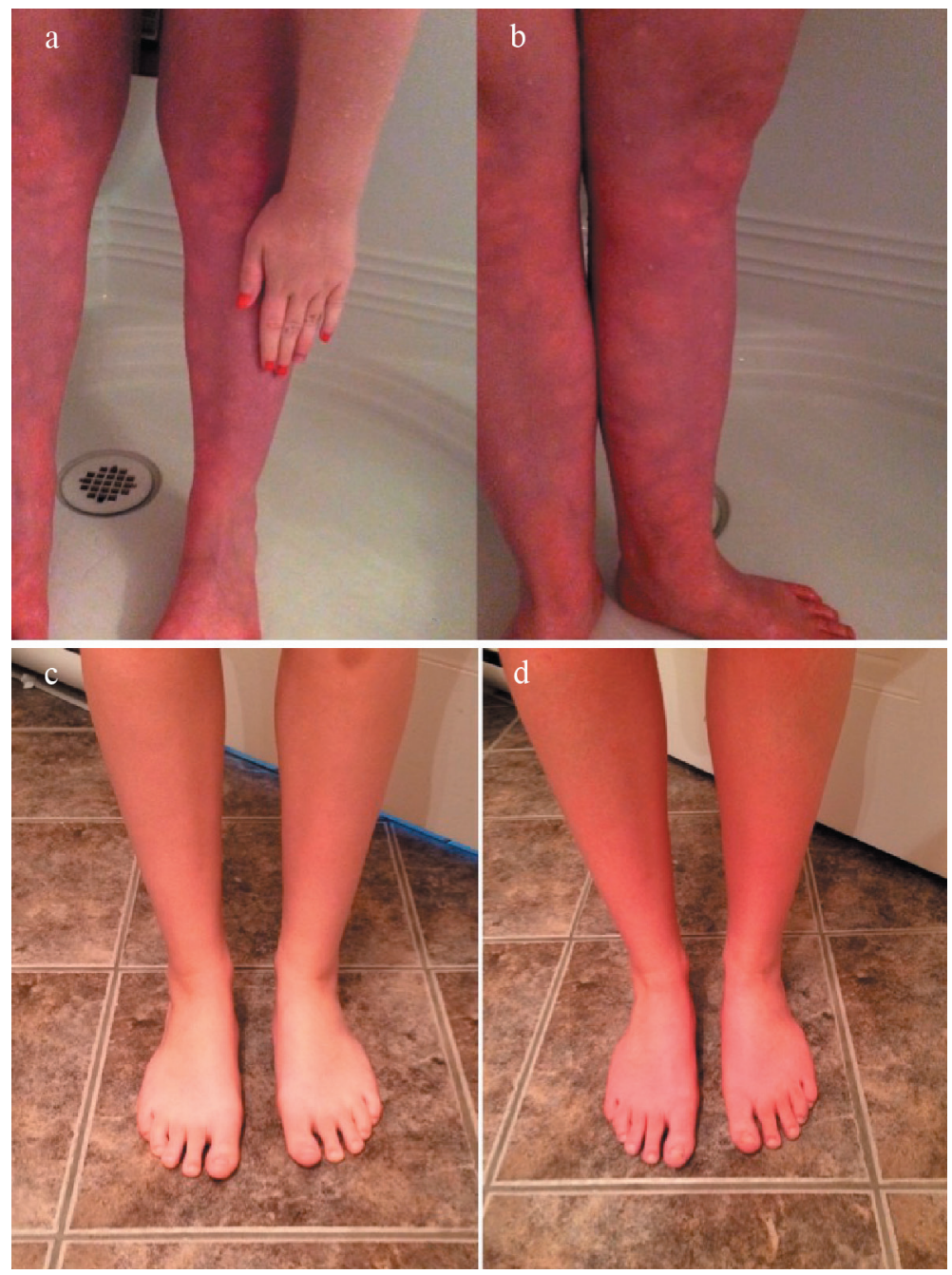

Figure 1. Dependent acrocyanosis in POT patients. $(a, b)$ The legs of the first case adolescent with POTS. The patient has significant red-blue discoloration of her legs extending up to the knees (a, b), compared to her arm (a), while standing. (c, d) The legs of the second case adolescent with POTS before (c) and after (d) staying in a standing position. The patient has significant red discoloration of her legs while standing.

to eight times per day and lasted between 2 to $15 \mathrm{~min}$. She also experienced dizziness with position change and lower limbs discoloration (Fig. 1c, d) when standing for a long period. She was known for having benign hypermobility, which limited her from performing competitive cheerleading. The physical exam was normal. Complete blood panel, electrocardiogram and cardiac ultrasound were all within normal limits. Holter measurement showed a sinus rhythm with a rate ranging from 53 to $164 \mathrm{bpm}$. A clinical diagnosis of POTS was then made. Non-pharmacological treatment was recommended. Propranolol was started after 6 weeks with positive results.

\section{Discussion}

POTS is characterized by a highly variable constellation of symptoms who all have in common dysautonomia symptoms. The pathopysiology remains unclear, but is likely to be mul- tifactorial. "Moderate autonomic dysfunction, increased sympathetic tone, severe deconditioning, inadequate venous return or excessive blood venous pooling" have all been cited as potential pathophysiologic explanations to the symptomatology [4]. A possible classification according to pathophysiology has been described: hyperadrenergic, hypovolemic and deconditioned, neuropathic and POTS with joint hypermobility [6].

POTS has been associated with different clinical conditions such as irritable bowel syndrome, hypermobility syndrome, vascular compression syndrome and chronic fatigue syndrome, to name a few $[4,6]$. It can present with a different symptomatology. The condition is often triggered by an illness, a prolonged recovery period, or an extreme athletic activity $[3,5,6]$. A recent review of literature examined the relationship between the human papillomavirus (HPV) vaccine and POTS, which is an association that was reported in a few case reports, and found no evidence that the HPV vaccine was a trigger for POTS [8]. 
This entity is important for pediatricians to recognize because early diagnosis and intervention can prevent significant functional consequences as well as reduce unnecessary investigations. In both our cases, the adolescents had dependant acrocyanosis associated with a constellation of non-specific symptoms, causing a delay in diagnosis. The diagnosis of POTS can be challenging. Patients can present orthostatic and sympathetic symptoms, associated with fatigue, headache, chronic pain, dizziness, gastrointestinal dysfunction such as irritable bowel syndrome, altered temperature sensation, sleep disturbances, hypermobility, and extremity changes including discoloration or peripheral edema $[3,5,6,9]$. The clinical history and exam is particularly important because the diagnosis of POTS is made clinically. Diagnostic criteria have been proposed and include: chronicity, excessive postural tachycardia as HR increment of $\geq 40 \mathrm{bpm}$ or an absolute orthostatic HR $\geq 130 \mathrm{bpm}$ for adolescents $\leq 13$ years old and $\geq 120$ bpm for adolescents $\geq 14$ years old, within 5 min of head-up tilt $[3,9]$. However, no clear definition has been established as a guideline [4].

In our cases, both adolescents described lower limbs discoloration which was position-dependant, an indicator of autonomic dysfunction diagnosis and a strong physical feature in approximately $50 \%$ of patients with POTS [10]. The pathophysiology may be related to a reduction in cutaneous microvascular neuronal nitric oxide activity, which increases central and peripheral sympathetic excitation [11]. Thus, this sigh should strongly point to the evaluation of POTS.

The first line of treatment is non-pharmacological and includes hydration $>2 \mathrm{~L} /$ day, increased salt intake, $10-15 \mathrm{~cm}$ elevation of the head of the bed, gradual reconditioning with aerobic exercises, support stocking (30 mm Hg) and psychological support $[3,5,6,9]$. Non-pharmacological treatment has been approved by Food and Drug Administration in pediatric POTS [6]. Different medications have been tried such as propranolol and midodrine, which were both used with our patients. Pharmacological treatment should be used with caution and adapted to the symptomatology and tolerance of the patient [4]. Ivabradine is a novel heart rate lowering agent and is a reasonable option for patients who do not respond to other treatments [12]. An important aspect of treatment is adopting a family-oriented treatment approach, as the family's interpretation of the symptoms can affect the degree of disability [13].

Five years after initial treatment, overall, $86 \%$ of adolescents with POTS report resolved, improved, or just intermittent symptoms [14]. A recent prospective study at the Mayo clinic combining pharmacological and non-pharmacological interventions found improvement in $70 \%$ of the patients 1 year after treatment [4]. Overall, there is a positive outcome in most of the patients following treatment.

\section{Conclusions}

In conclusion, POTS can be identified clinically by the experienced clinician who can recognize its symptomatology. We state that dependant acrocyanosis can help guide towards a clinical diagnosis of POTS, which can present with a wide symptomatology, thus being difficult to be recognized. Recog- nizing acrocyanosis as a sign of POTS can help lower unnecessary testing and treatments for patients.

\section{Ethical Approval}

This article does not contain any studies with human participants or animals performed by any of the authors.

\section{Parental Approval}

Our manuscript does not contain data from an individual permitting the identification of such person; therefore, no parental approval is needed.

\section{Conflict of Interest}

The authors declare that they have no conflict of interest.

\section{Author Contributions}

Jane Abou-Diab wrote the manuscript, Dina Moubayed is the second author who updated the manuscript and reviewed it, Danielle Taddeo and Olivier Jamoulle reviewed the manuscript and Chantal Stheneur reviewed the manuscript and supervised the first and second author.

\section{Financial Support}

No financial support was granted for this case report. No grants were obtained.

\section{References}

1. Schondorf R, Low PA. Idiopathic postural orthostatic tachycardia syndrome: an attenuated form of acute pandysautonomia? Neurology. 1993;43(1):132-137.

2. Stewart JM, Gewitz MH, Weldon A, Munoz J. Patterns of orthostatic intolerance: the orthostatic tachycardia syndrome and adolescent chronic fatigue. J Pediatr. 1999;135(2 Pt 1):218-225.

3. Kizilbash SJ, Ahrens SP, Bruce BK, Chelimsky G, Driscoll SW, Harbeck-Weber C, Lloyd RM, et al. Adolescent fatigue, POTS, and recovery: a guide for clinicians. Curr Probl Pediatr Adolesc Health Care. 2014;44(5):108133.

4. Wells R, Spurrier AJ, Linz D, Gallagher C, Mahajan R, Sanders P, Page A, et al. Postural tachycardia syndrome: current perspectives. Vasc Health Risk Manag. 2018;14:111.

5. Pilcher TA, Saarel EV. A teenage fainter (dizziness, syncope, postural orthostatic tachycardia syndrome). Pediatr Clin North Am. 2014;61(1):29-43. 
6. Jarjour IT. Postural tachycardia syndrome in children and adolescents. Semin Pediatr Neurol. 2013;20(1):18-26.

7. Ojha A, Chelimsky TC, Chelimsky G. Comorbidities in pediatric patients with postural orthostatic tachycardia syndrome. J Pediatr. 2011;158(1):20-23.

8. Butts BN, Fischer PR, Mack KJ. Human papillomavirus vaccine and postural orthostatic tachycardia syndrome: a review of current literature. J Child Neurol. 2017;32(11):956-965.

9. $\mathrm{Xu} \mathrm{WR}$, Jin HF, Du JB. Pathogenesis and Individualized Treatment for Postural Tachycardia Syndrome in Children. Chin Med J (Engl). 2016;129(18):2241-2245.

10. Raj SR. Postural tachycardia syndrome (POTS). Circulation. 2013;127(23):2336-2342.

11. Stewart JM, Medow MS, Minson CT, Taneja I. Cutaneous neuronal nitric oxide is specifically decreased in postural tachycardia syndrome. Am J Physiol Heart Circ Physiol. 2007;293(4):H2161-2167.

12. Gee ME, Watkins AK, Brown JN, Young EJA. Ivabradine for the treatment of postural orthostatic tachycardia syndrome: a systematic review. Am J Cardiovasc Drugs. 2018.

13. Keating EM, Antiel RM, Weiss KE, Wallace D, Antiel SJ, Fischer PR, Junghans-Rutelonis AN, et al. Parental perceptions of pediatric pain and POTS-related disability. Clin Pediatr (Phila). 2017;56(13):1185-1192.

14. Bhatia R, Kizilbash SJ, Ahrens SP, Killian JM, Kimmes SA, Knoebel EE, Muppa P, et al. Outcomes of adolescentonset postural orthostatic tachycardia syndrome. J Pediatr. 2016;173:149-153. 